\title{
Observations of Interplanetary Scintillation (IPS) Using the Mexican Array Radio Telescope (MEXART)
}

\author{
J.C. Mejia-Ambriz • P. Villanueva-Hernandez • \\ J.A. Gonzalez-Esparza • E. Aguilar-Rodriguez • \\ S. Jeyakumar
}

Received: 8 December 2009 / Accepted: 24 April 2010 / Published online: 29 May 2010

(C) The Author(s) 2010. This article is published with open access at Springerlink.com

\begin{abstract}
The Mexican Array Radio Telescope (MEXART) consists of a $64 \times 64$ (4096) full-wavelength dipole antenna array, operating at $140 \mathrm{MHz}$, with a bandwidth of $2 \mathrm{MHz}$, occupying about 9660 square meters $(69 \mathrm{~m} \times 140 \mathrm{~m})$ (http://www.mexart.unam.mx). This is a dedicated radio array for Interplanetary Scintillation (IPS) observations located at latitude $19^{\circ} 48^{\prime} \mathrm{N}$, longitude $101^{\circ} 41^{\prime} \mathrm{W}$. We characterize the performance of the system. We report the first IPS observations with the instrument, employing a Butler Matrix $(\mathrm{BM})$ of $16 \times 16$ ports, fed by 16 east - west lines of 64 dipoles ( $1 / 4$ of the total array). The BM displays a radiation pattern of 16 beams at different declinations (from -48 , to +88 degrees). We present a list of 19 strong IPS radio sources (having at least $3 \sigma$ in power gain) detected by the instrument. We report the power spectral analysis procedure of the intensity fluctuations. The operation of MEXART will allow us a better coverage of solar wind disturbances, complementing the data provided by the other, previously built, instruments.
\end{abstract}

Keywords Interplanetary scintillations · Interplanetary shocks · CMEs · Remote sensing · Radiotelescopes

\section{Introduction}

The phenomenon called Interplanetary Scintillation (IPS) was discovered by Hewish, Scott, and Wills (1964). The IPS is the random intensity variation of a cosmic radio source of

Remote Sensing of the Inner Heliosphere

Guest Editors: M.M. Bisi, and A.R. Breen.

J.C. Mejia-Ambriz · P. Villanueva-Hernandez

Posg. Ciencias de la Tierra, Universidad Nacional Autonoma de Mexico, Ciudad Universitaria, Mexico DF 04510, Mexico

J.C. Mejia-Ambriz · P. Villanueva-Hernandez · J.A. Gonzalez-Esparza ( $₫)$ · E. Aguilar-Rodriguez MEXART, Inst. de Geofisica, Unidad Michoacán, Universidad Nacional Autonoma de Mexico, Tzintzuntzan 310, Col. Vista Bella, Morelia, Michoacan 58098, Mexico

e-mail: americo@geofisica.unam.mx

S. Jeyakumar

Dept. de Astronomia, Universidad de Guanajuato, AP 144, Guanajuato CP, 36000, Mexico 

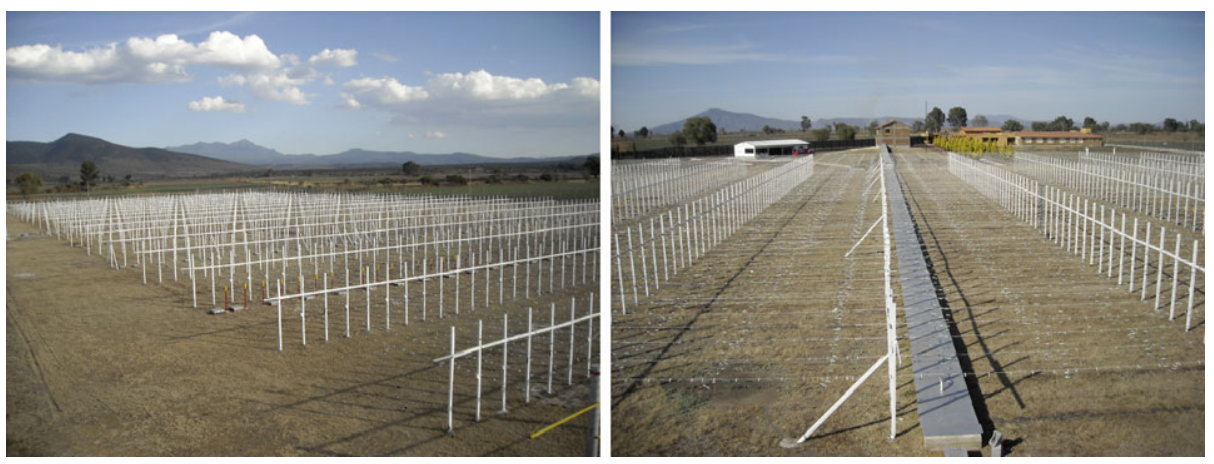

Figure 1 Pictures of the MEXART facilities. (Left) The antenna, showing the ensemble of the 4096 dipoles. (Right) Facilities at the site: antenna, control room, laboratories, staff offices and guest house for students and visitors.

small diameter $(<2$ arc sec) caused by the scattering of the wave front as it propagates through random fluctuations in the refractive index of the turbulent interplanetary medium. These phase distortions of the radio wave front are related to small-scale electron density inhomogeneities $\left(\Delta n_{\mathrm{e}}\right)$ in the interplanetary plasma that produce a diffraction pattern in the plane of the observer and create intensity fluctuations (scintillations) as the diffraction pattern is convected across the antenna by the solar wind (Ananthakrishnan and Kaufman, 1980).

The IPS technique assumes that these electron density fluctuations $\left(\Delta n_{\mathrm{e}}\right)$ are proportional to density variations in the solar wind. Significant enhancements in solar-wind density above what may be expected in the background solar wind is likely to be related with two general types of solar-wind disturbances:

i) The region around a stream interface between a fast solar-wind stream interacting with a slow ambient wind (stream interaction region).

ii) The density enhancement associated with solar transient events such as Interplanetary counterparts of Coronal Mass Ejections (ICMEs) and their associated shocks.

We can use IPS observations to identify large-scale solar-wind perturbations propagating from the Sun to $1 \mathrm{AU}$ (Hewish and Bravo, 1986). A network of IPS stations distributed along different longitudes can help to track the evolution of solar-wind perturbations.

The aim of this paper is to report the first observations of IPS sources detected by the Mexican Array Radio Telescope (MEXART). We describe the array's characteristics, the instrument current sensitivity, the initial list of IPS sources, and the power spectral analysis of the intensity fluctuations.

\section{Description of the Array}

MEXART is located in Coeneo, Michoacan, at a latitude of $19^{\circ} 48^{\prime} \mathrm{N}$, and a longitude of $101^{\circ} 41^{\prime} \mathrm{W}$. Figure 1 shows two pictures of the MEXART antenna and the facilities at the site. MEXART has an operation frequency of $139.65 \mathrm{MHz}$. The array has 64 parallel east west $(\mathrm{E}-\mathrm{W})$ rows and each row has 64 dipoles. Every $\mathrm{E}-\mathrm{W}$ row can be considered as a uniform linear array of 64 elements with the same amplitude and phase having a length of $64 \lambda$. In total, the array has $64 \times 64=4096$ elements occupying $(69 \mathrm{~m} \times 140 \mathrm{~m}) 9660$ square meters. 


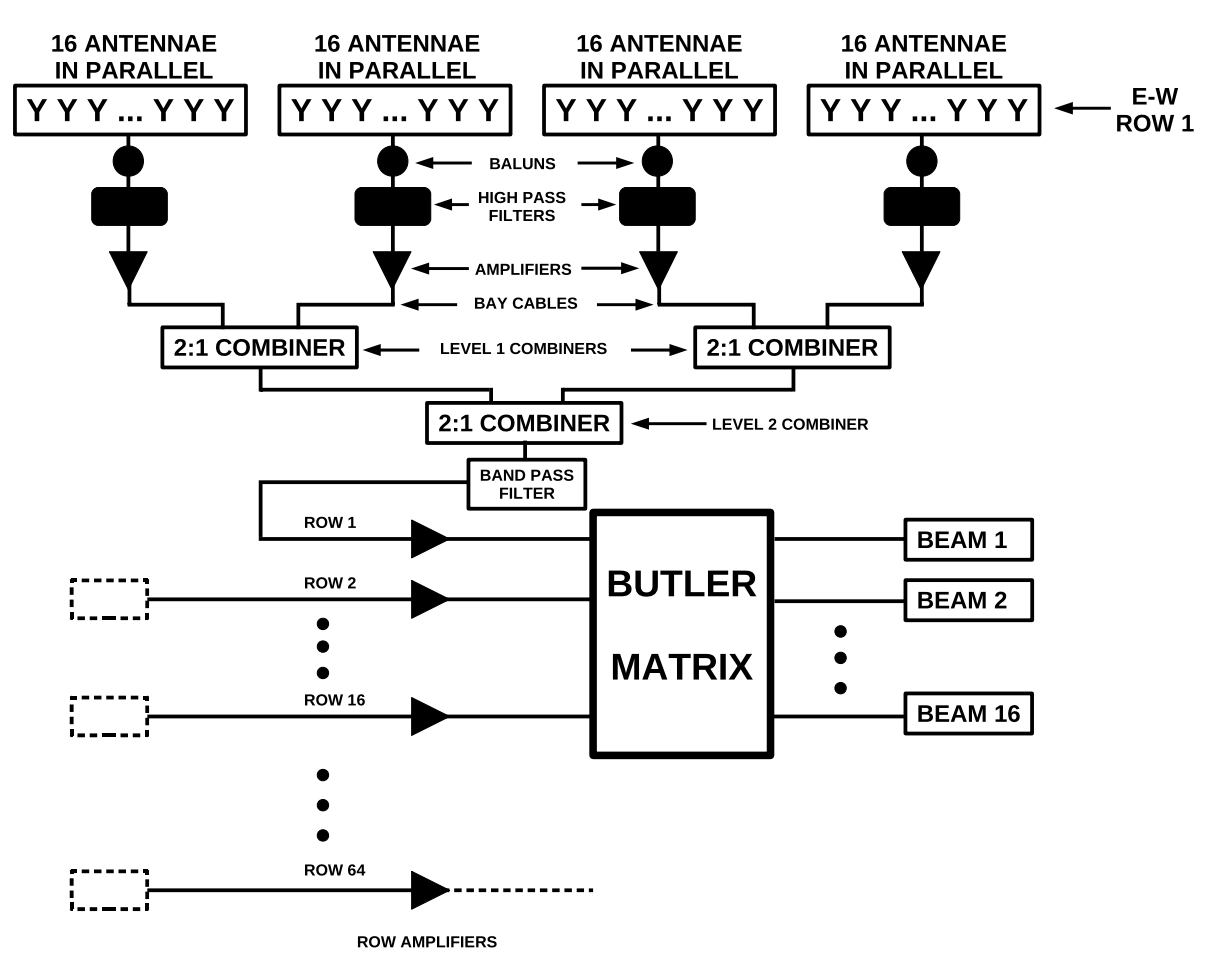

Figure 2 Diagram of MEXART antenna. The array has 64 parallel E-W rows and each row has 64 dipoles which are divided in four sections of 16 dipoles. The signal from the dipoles are combined in several stages including: two filtering, two amplification and two combination steps. In this work we report observations using $16 \mathrm{E}-\mathrm{W}$ rows and a Butler Matrix of $16 \times 16$ ports.

Figure 2 shows a block diagram of the array. The basic components are: antennae (dipoles), baluns, high-pass and band-pass filters, amplifiers, combiners, and a $16 \times 16$ Butler Matrix (BM). The basic element of the array is the full-wavelength dipole $(\lambda=2.14 \mathrm{~m})$. Each $\mathrm{E}-\mathrm{W}$ row of 64 dipoles is divided into four sections. Each section is composed of a 16 dipole ensemble connected to a parallel transmission line. The signal of each section of 16 dipoles $(413 \Omega)$ is coupled with the co-axial transmission line $(50 \Omega)$ through the balun. The signal of each section is filtered (high-pass filter $f>67 \mathrm{MHz}$ ) and amplified ( $20 \mathrm{~dB}$ ). A low noise front-end amplifier is used for each module in a row. The amplified signals of each section are added vectorially by a 2:1 power combiner. At the end, each $\mathrm{E}-\mathrm{W}$ row has two amplification steps and two combination steps before sending the signal to the back end (the final band-pass filter of $15 \mathrm{MHz}$ is centered at $139.65 \mathrm{MHz}$ ). The rows are added together using a $\mathrm{BM}$ in the control room. The $\mathrm{BM}$ combines the signals of $16 \mathrm{E}-\mathrm{W}$ rows to produce a radiation pattern of 16 beams pointing at different declinations. The BM was designed at the Giant Metrewave Radio Telescope (GMRT) - National Centre for Radio Astrophysics (NCRA) (Shankarsubramanian and Sureshkumar, 2003) and it was assembled and tested at the MEXART site (Carrillo, 2007). Further details of the antenna array, amplification and combination systems, and receiver can be found in González-Esparza et al. (2004).

In 2004-2005 we initiated the calibration testings of the antenna radiation pattern using $\mathrm{E}-\mathrm{W}$ rows in a full power configuration to observe transits of the Sun and strong cosmic radio sources (González-Esparza et al., 2005, 2006). Table 1 summarizes the instrument 
Table 1 MEXART technical characteristics.

\begin{tabular}{ll}
\hline Observing frequency & $139.65 \mathrm{MHz}$ \\
\hline $\begin{array}{l}\text { Antenna } \\
\text { basic element }\end{array}$ & $\begin{array}{l}\text { plane array }(64 \times 64) \\
\text { full-wavelength dipole } \\
(\lambda=2.14 \mathrm{~m})\end{array}$ \\
Number of E-W rows & 64, each row has 64 \\
& elements \\
total physical area & $9660 \mathrm{~m}^{2}$ \\
Receiver & \\
band width & \\
integration time & $2.0 \mathrm{MHz}$ \\
local oscillator & $47 \mathrm{~ms}$ \\
intermediate frequency & $129 \mathrm{MHz}$ \\
sampling rate & $10.65 \mathrm{MHz}$ \\
\end{tabular}

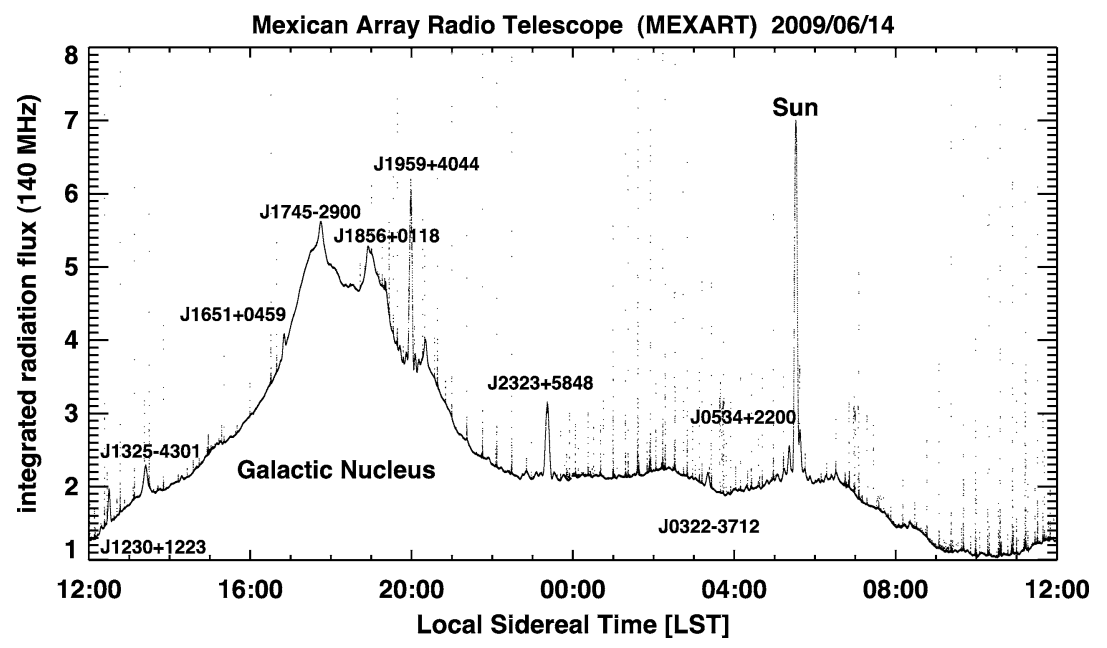

Figure 3 Integrated radiation flux at $140 \mathrm{MHz}$ of the transit of the Galaxy detected by MEXART on 14 June 2009.

characteristics. In this work, we report observations using $16 \mathrm{E}-\mathrm{W}$ rows $(1 / 4$ of the antenna, covering a physical area of about $2400 \mathrm{~m}^{2}$ ) and a BM of $16 \times 16$ ports.

MEXART is a transit instrument that uses the Earth's rotation to track the sky. We record on a daily basis the integrated radiation flux of the Galaxy at $140 \mathrm{MHz}$ through the 16 beams generated by the BM. Figure 3 shows an example of the radiation flux of the Galaxy in June 2009 as detected by one of the beams. The plot shows the transit of some of the strongest radio sources such as $\mathrm{J} 1230+1223$, J1651+0459, J1745-2900, J1856+0118, J2323+5848, J0534+2200 and the Sun among others. This daily scan is reported on real time at the observatory website (http://www.mexart.unam.mx). 
Table 2 Expected telescope sensitivity and confusion limit for various configurations.

\begin{tabular}{lclcc}
\hline Configuration & $\begin{array}{l}A_{\text {eff }} \\
{\left[\mathrm{m}^{2}\right]}\end{array}$ & $\begin{array}{l}\text { Sensitivity } \\
{\left[\mathrm{K} \mathrm{Jy}^{-1}\right]}\end{array}$ & $\begin{array}{l}\Delta S_{\text {rms }} \\
{[\mathrm{Jy}]}\end{array}$ & $\begin{array}{l}\text { Confusion limit } \\
{[\mathrm{Jy}]}\end{array}$ \\
\hline 1 row & 167.9 & 0.06 & 25 & 137 \\
16 rows & $1644^{*}$ & 0.59 & 2.5 & 16.5 \\
Full array & $6576^{*}$ & 2.38 & 0.6 & 5.4 \\
\hline
\end{tabular}

${ }^{*}$ Calculated by assuming an efficiency factor of 0.7 .

\section{Sensitivity of the Array}

The minimum flux of a source that can be observed with the radiotelescope depends on the instrument's characteristics and the random noise generated by the electronic components, cables, etc., along the path of the signal. In addition, the estimated scintillation index, which depends on the total flux of the source, will have an uncertainty depending on the faint radio sources in the beam of the telescope, the so-called confusion limit (Condon, 1974). In this section, we calculate the expected sensitivity of MEXART, the confusion limit, the number of IPS sources, and the observable characteristics of the IPS map of the sky.

The sensitivity of the telescope $\left(\mathrm{K} \mathrm{Jy}^{-1}\right)$ for single polarization is $A_{\text {eff }} / 2 k$, where $A_{\text {eff }}$ is the effective area, and $k$ is the Boltzmann constant. The rms of the noise fluctuations is given by:

$$
\Delta T_{\mathrm{rms}}=\frac{T_{\mathrm{sys}}}{\sqrt{\beta \tau}},
$$

where the system temperature, $T_{\text {sys }}$ is the sum of receiver temperature $T_{\mathrm{R}}$ and the background sky temperature $T_{\text {sky }}$ (Krauss, 1986). For a noise figure of the front-end amplifier of $2.9 \mathrm{~dB}$, we estimate the receiver temperature $T_{\mathrm{R}}=275 \mathrm{~K}$. In general, the integrated radiation flux of the sky background at $140 \mathrm{MHz}$ varies from one region to another, therefore, assuming a mean value of $200 \mathrm{~K}$, the $T_{\text {sys }}=475 \mathrm{~K}$. For a bandwidth, $\beta$, of $2 \mathrm{MHz}$, and integration time, $\tau$, of 50 milliseconds, $\sqrt{\beta \tau}$ is 316.2 .

We calculate $A_{\text {eff }}$ of the array as follows. For 1 row of 64 dipoles, we estimated the effective collecting area of each $\lambda$ dipole using $\mathrm{A}_{\text {eff }}=\left(\lambda^{2} \cdot G\right) / 4 \pi$ (where $G$ is the effective gain). The gain of each dipole will be affected by coupling effects between dipoles, so we obtain $G$ of a dipole by dividing the total directivity of the $\mathrm{E}-\mathrm{W}$ array (calculated using the program PCAAD 5.0; Pozar, 2002) by the total number of dipoles. The $G$ of single dipole in an $\mathrm{E}-\mathrm{W}$ array of 64 dipoles (1 row) including the coupling effects, is 7.14, and $A_{\text {eff }}$ of each dipole is $2.61 \mathrm{~m}^{2}$ (Carrillo, 2007). For several $\mathrm{E}-\mathrm{W}$ rows of 64 dipoles, we need also to take into account the coupling effects between the rows by an efficiency factor, which we estimated based on observations of the Crab Nebula (J0534+2200). Therefore, we used an efficiency of about $70 \%$ for the calculation of $A_{\text {eff }}$. In Table 2 we present an estimate of the array's $A_{\text {eff }}$, sensitivity, and the minimum detectable flux density $\left(\Delta S_{\text {rms }}=\left(2 k / A_{\text {eff }}\right) \Delta T_{\text {rms }}\right)$ for different array configurations of $\mathrm{E}-\mathrm{W}$ rows. 


\subsection{Confusion Limit}

The confusion noise due to faint radio sources within the beam depends on the beam size. The confusion limit is calculated following Condon (1974),

$$
S_{\mathrm{c}}=\left(\frac{q^{3-\gamma}}{3-\gamma}\right)^{\frac{1}{1-\gamma}}\left(n_{0} \Omega_{\mathrm{b}}\right)^{\frac{1}{1-\gamma}},
$$

where $\gamma$ and $n_{0}$ are defined as, $n(s) d s=n_{0} s^{\gamma} d s$. Here $n(s)$ is the differential source count. The effective telescope beam is

$$
\Omega_{\mathrm{b}}=\frac{\pi}{4} \frac{\theta_{1} \theta_{2}}{(\gamma-1) \ln (2)} .
$$

The beam widths in two perpendicular directions are denoted by $\theta_{1}$ and $\theta_{2}$. The factor $q$ is usually taken to be 5 . We use the source count at $178 \mathrm{MHz}$ from the Cambridge survey given as $\gamma=2.3$ and $n_{0}=1.0 \times 10^{3}$ sources $\mathrm{sr}^{-1}$ (the operating frequency of MEXART is close enough). Using these values,

$$
S_{\mathrm{c}}=4.1454\left(\theta_{1}^{\prime \prime} \theta_{2}^{\prime \prime}\right)^{1 / 1.3} \mathrm{Jy} .
$$

The confusion limit for different configurations are also listed in Table 2.

\subsection{Observable Sources}

For detection at the 3 sigma $(\sigma)$ level with a $2 \mathrm{MHz}$ bandwidth, the detection limit is about $1.8 \mathrm{Jy}$. For a spectral index of 0.75 , this detection limit at $178 \mathrm{MHz}$ is smaller by a factor of 1.137. This yields a detection limit of $1.6 \mathrm{Jy}$ at $178 \mathrm{MHz}$. The number of sources brighter than $1.6 \mathrm{Jy}$ at $178 \mathrm{MHz}$ is 4900 (obtained using the NASA/IPAC Extragalactic Database (NED) http://nedwww.ipac.caltech.edu/index.html). Restricting the scintillation targets to elongation angles between 45 and 15 degrees, the fraction of sky covered is about 0.108 . Thus, the number of sources that can be easily detected by MEXART using the IPS technique in a day is around 490 when fully operational.

\subsection{IPS of Radio Sources}

At the operating frequency of $140 \mathrm{MHz}$, the angular size of the source that will scintillate is less than two arc sec. The scintillation index is defined as: $m=\Delta S_{\mathrm{rms}} / S_{\mathrm{T}}$, where $\Delta S_{\mathrm{rms}}$ is the scintillating flux and $S_{\mathrm{T}}$ is the total flux of the source. For sources detected at $N \sigma$ level, the lower limit to $m$ is $1 / N$. On the other hand, $m$ of a point source decreases as the elongation angle $(\epsilon)$ increases. As shown in Figure 4, $m$ is related to $\epsilon$ as $m \propto \sin (\epsilon)^{-1.75}$ (Manoharan et al., 1995). A lower limit on $m$ sets an upper limit detection level. Table 3 presents the lower limit to $m$ and the corresponding upper limit to $\epsilon$ for different detection limits for the full array.

\subsection{Scintillation Map and Observing Strategy}

Figure 5 shows the regions (drawn as circles) set by the upper limits to $\epsilon$. The minimum required $\mathrm{S} / \mathrm{N}$ of the sources are also quoted for each circle. The strong scattering region is shown in grey (inner circle). Assuming that most of the sources are close to the detection limit, the number of sources that would fall within each zone are given in Table 3 . This is calculated assuming that $\epsilon$ less than $15^{\circ}$ are not observable due to strong scattering. 
Figure 4 Variation of scintillation index with solar elongation angle.

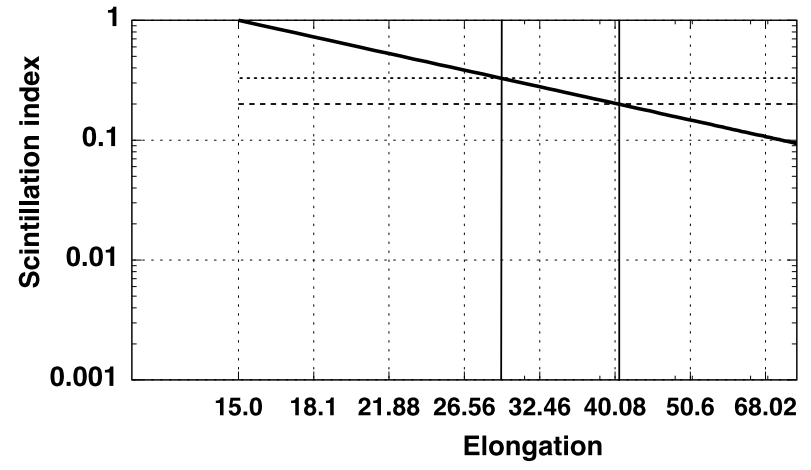

Figure 5 Regions of the sky with the detection/scintillation index limits. The horizontal axis is the elongation angle $(\epsilon)$ and the circles the scintillation regions set by the upper limits (see text). The Sun is located at the center.

Table 3 Table of IPS-zone and the number of sources.

\begin{tabular}{lllll}
\hline Observing limit & $m$-limit & $\begin{array}{l}\epsilon \text {-limit } \\
\text { degree }\end{array}$ & \multicolumn{2}{c}{ Number of sources } \\
\hline 1.8 Jy $(3 \sigma)$ & 0.33 & 29 & 4900 & IPS-zone \\
3.0 Jy $(5 \sigma)$ & 0.2 & 40 & 3000 & 310 \\
6.0 Jy $(10 \sigma)$ & 0.1 & 75 & 826 & 340 \\
\hline
\end{tabular}

\section{List of IPS Sources}

In order to set up the IPS observations at MEXART we used the IPS source lists reported in the first Cambridge's catalogue (Hewish, Scott, and Wills, 1964), the Ooty Radio Telescope (ORT) (Manoharan, private communication) and the Solar-Terrestrial Environment Laboratory (STEL) (Tokumaru, private communication). Table 4 shows the list of the first strong IPS sources detected by MEXART with the present configuration, using the $16 \times 16 \mathrm{BM}$. The first column is the name of the source. The second column is an estimated interpolated radiation flux at $140 \mathrm{MHz}$, using data points in the range of $\sim 30$ to $700 \mathrm{MHz}$ taken from the NASA/IPAC Extragalactic Database (NED) (http://nedwww.ipac.caltech.edu/index.html), 
Table 4 List of IPS sources detected at MEXART with the current system: name of the source, interpolated radiation flux at $140 \mathrm{MHz}$, IPS catalogue ( $\mathrm{C}=3 \mathrm{C}$ Cambridge, $\mathrm{O}=$ Ooty

Radio Telescope,

$\mathrm{S}=$ Solar-Terrestrial

Environment Laboratory), and signal-to-noise ratio $(\mathrm{S} / \mathrm{N})$ in $\sigma$ detected by the instrument.

\begin{tabular}{|c|c|c|c|}
\hline $\begin{array}{l}\text { Radio } \\
\text { source }\end{array}$ & $\begin{array}{l}\text { Flux } 140 \mathrm{MHz} \\
\text { (Jy) }\end{array}$ & $\begin{array}{l}\text { IPS } \\
\text { cat. }\end{array}$ & $\mathrm{S} / \mathrm{N}$ \\
\hline $\mathrm{J} 0108+1320$ & 65 & $\mathrm{O}$ & 4.3 \\
\hline $\mathrm{J} 0137+3309$ & 68 & $\mathrm{O}, \mathrm{S}, \mathrm{C}$ & 3.4 \\
\hline $\mathrm{J} 0319+4130$ & 66 & $\mathrm{O}, \mathrm{S}$ & 7.1 \\
\hline J0322-3712 & 383 & $\mathrm{O}$ & 13.4 \\
\hline $\mathrm{J} 0408+4300$ & 30 & $\mathrm{O}$ & 3.8 \\
\hline J0418+3801 & 65 & $\mathrm{O}$ & 4.0 \\
\hline J0504+3806 & 87 & $\mathrm{O}$ & 7.8 \\
\hline $\mathrm{J} 0813+4813$ & 77 & $\mathrm{O}, \mathrm{S}, \mathrm{C}$ & 6.7 \\
\hline $\mathrm{J} 1008+0730$ & 30 & $\mathrm{O}, \mathrm{S}, \mathrm{C}$ & 3.1 \\
\hline $\mathrm{J} 1149+1247$ & 19 & $\mathrm{O}, \mathrm{S}$ & 3.6 \\
\hline $\mathrm{J} 1229+0203$ & 104 & $\mathrm{O}, \mathrm{S}, \mathrm{C}$ & 12.9 \\
\hline J1254-1233 & 39 & $\mathrm{O}$ & 6.6 \\
\hline J1311-2216 & 46 & $\mathrm{O}, \mathrm{S}$ & 4.6 \\
\hline $\mathrm{J} 1411+5212$ & 100 & $\mathrm{C}$ & 5.8 \\
\hline $\mathrm{J} 1419+0628$ & 71 & $\mathrm{O}, \mathrm{S}, \mathrm{C}$ & 4.2 \\
\hline $\mathrm{J} 1504+2600$ & 75 & $\mathrm{O}$ & 5.2 \\
\hline $\mathrm{J} 1651+0459$ & 463 & $\mathrm{O}$ & 17.1 \\
\hline $\mathrm{J} 2155+3800$ & 55 & $\mathrm{O}$ & 7.3 \\
\hline $\mathrm{J} 2245+3941$ & 67 & $\mathrm{O}$ & 7.0 \\
\hline
\end{tabular}

the SIMBAD astronomical database (http://simbad.u-strasbg.fr/simbad/) or Edge et al. (1959), and the fitting curve was obtained with a least-square polynomial function of IDL routine. The third column shows the reference where the source is found, label ' $\mathrm{O}$ ', ' $\mathrm{S}$ ', and/or ' $\mathrm{C}$ ' indicating if the source is in ORT, STEL, and/or IPS Cambridge's list respectively. The fourth column is the average signal-to-noise ratio $(\mathrm{S} / \mathrm{N})$ detected by the instrument. The 19 sources present a $\mathrm{S} / \mathrm{N}>3$.

The radio sources in Table 4 are located in a declination range between -37 to 52 degrees. Figure 6 shows the radio sky background contours at $250 \mathrm{MHz}$ (Krauss, 1986) and the location of the IPS sources in Table 4. The contours indicate the equivalent brightness temperature of the sky background, and the dashed line the MEXART's zenith declination $(20 \mathrm{deg})$. The figure shows the relative route of the Sun through the radio sky along the year, from the March equinox ( 0 hours, $0 \mathrm{deg}$ ), passing across the June solstice (6 hours, 23.5 deg), September equinox (12 hours, $0 \mathrm{deg}$ ) and December solstice (18 hours, $-23.5 \mathrm{deg}$ ). This figure allow us to qualitatively estimate the epochs of low or high elongation angle for these sources.

\section{Spectral Analysis}

The most important physical magnitude for the IPS analysis is the time series of the fluxintensity fluctuations on the source transit. The IPS is a stochastic process, so the solar-wind information cannot be obtained from the study of the time series itself but from the statistical properties in the temporal and spatial domains.

The Fresnel propagation function represents the interference effects that produce the intensity scintillation. This filter relates the fluctuations in electron density $n_{\mathrm{e}}$ across the line 


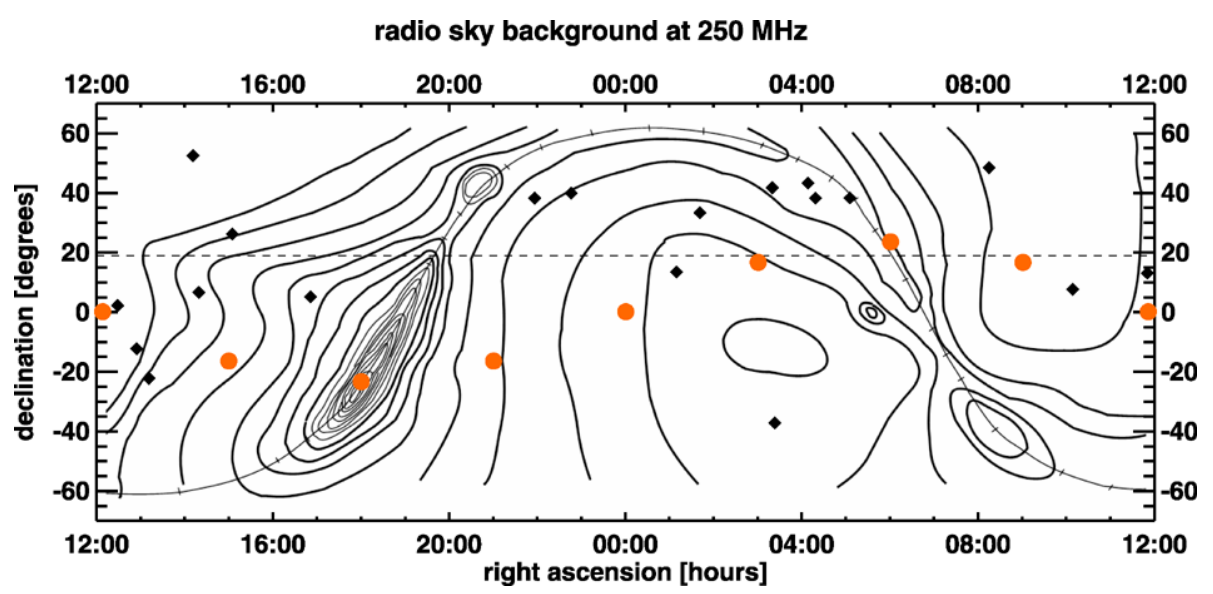

Figure 6 Location of the IPS sources detected by MEXART (solid diamonds) with respect to the background radio sky. The contours indicate the equivalent brightness temperature of the sky background at $250 \mathrm{MHz}$ (adapted from Krauss, 1986). Different locations of the Sun (grey solid circles) are also indicated along the year with respect to the radio sky.

of sight (los) with the flux-intensity fluctuations on the observing plane (Manoharan, 1991; Oberoi, 2000). If we can assume that the scattering is mainly produced by a thin screen at the $P$-point ( $P$ is the closest point to the Sun along the los to the source) and the remainder of the los is neglected, then the Fresnel propagation function becomes:

$$
F_{f}=\sin ^{2}\left(\frac{q^{2} \lambda z}{4 \pi}\right),
$$

where $z$ is the distance to the $P$-point, $\lambda$ is the observation wavelength, and $q$ is the characteristic frequency in the frequency spatial domain. This former is related to the solar-wind speed $(V)$ and the temporal frequency $(f)$ as follows:

$$
q=2 \pi f / V .
$$

Figure 7 shows the solution of the Fresnel propagation function (Equation (4)) for the MEXART operation frequency $(\lambda=2.14 \mathrm{~m})$, an elongation angle $\epsilon=20^{\circ}$, and a solarwind speed of $400 \mathrm{~km} \mathrm{~s}^{-1}$. After the first maxima of the Fresnel oscillations the intensityfluctuation spectrum shows a sharp fall in power. This shape is known as the Fresnel knee.

\subsection{MEXART Observations}

The first MEXART observations of IPS sources were obtained using $16 \mathrm{E}-\mathrm{W}$ rows of 64 dipoles each and a BM of $16 \times 16$ ports, the receiver has a bandwidth of $2.0 \mathrm{MHz}$, a time constant of $47 \mathrm{~ms}$, and the data was sampled every $20 \mathrm{~ms}$. Figure 8 shows one of the transits of J0137+3309 detected by MEXART in April 2009, where the source had an elongation angle $\epsilon \approx 21^{\circ}$. During the source transit the rms increases show the signature of the IPS phenomenon ( $\sigma$ in the on-source record is 0.035 , while in the off-source record is 0.012 ). There was a strong interference (probably related with a satellite signal), which was removed for the spectral analysis. 
Figure 7 Fresnel propagation function, $\sin ^{2}\left(q^{2} z / 2 k\right)$, in a $\log -\log$ scale for $\lambda=2.14 \mathrm{~m}$, $\epsilon=20^{\circ}$, and $V=400 \mathrm{~km} \mathrm{~s}^{-1}$.

Figure 8 Transit of J0137+3309 (3C048 in the 3C Cambridge catalogue) detected by MEXART on 22 April 2009.
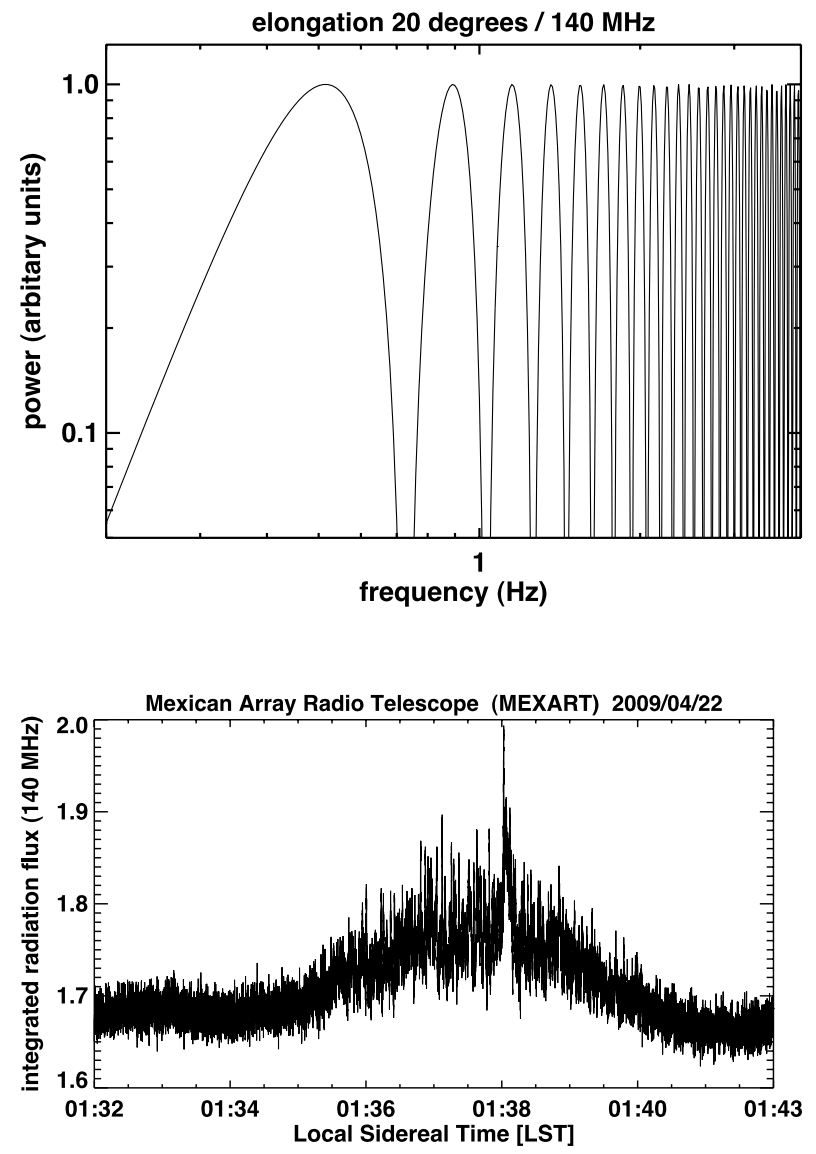

The procedure to perform the spectral analysis of the intensity fluctuations is based on the one developed at ORT (Rao, Bhandari, and Ananthakrishnan, 1974; Manoharan, 1991; Oberoi, 2000). This procedure can be summarized as follows: $i$ ) we take an on-source recording of 52 seconds; $i$ ) we subtract from the record running means over 10 seconds; iii) we taper the data points at the beginning and the end of the record to minimize the edge effects; $i v$ ) we apply a Fast Fourier Transform to the record; the power spectrum is obtained by squaring the amplitude of the Fourier coefficients. These procedures are carried out on on-source record and on off-source record separately. Finally, v) the off-source spectrum, or the spectrum of the receiver noise, is subtracted from the on-source spectrum to give the true spectrum of the intensity fluctuations; and $v i$ ) we correct the low-frequency region of the final spectrum $(f \leq 0.3 \mathrm{~Hz})$ by extrapolating a fitting second-degree polynomial to the next forty points of the corrected spectrum. Figure 9 shows the power spectra analysis of the intensity fluctuations of $J 0137+3309$. The plot on the left shows the power spectra on a lineal scale, whereas the plot of the right on logarithmic scale. Both show clearly the shape of the Fresnel knee in the frequency range associated with the IPS phenomenon. The first minimum occurs at around $1 \mathrm{~Hz}$. The rms on the on-source record is 0.0354 and on the off-source record is 0.0122. Although the power spectra in Figure 9 show the Fresnel knee, there is not enough spectrum to show the usual power law variation beyond that. The reason 

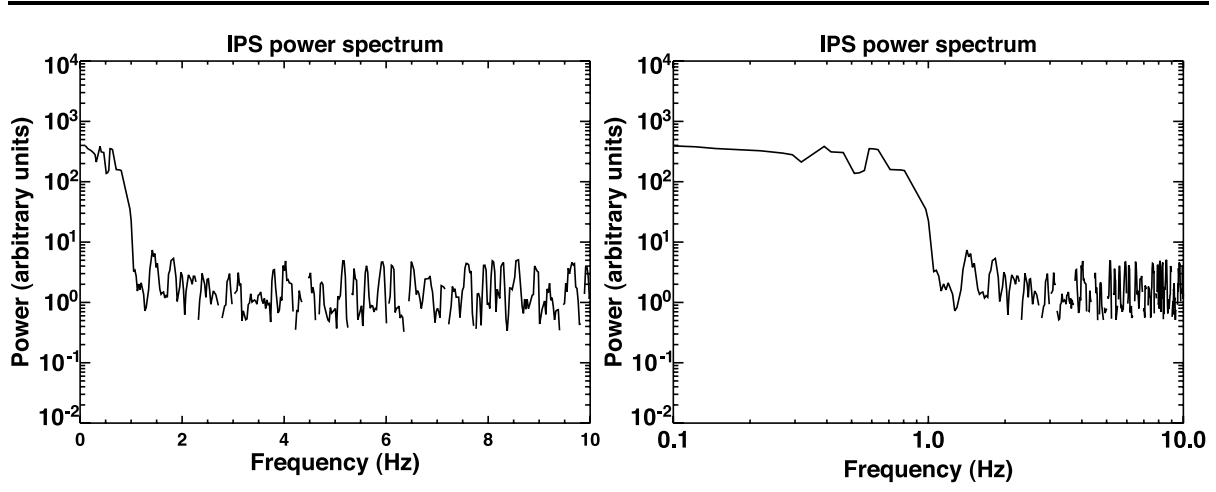

Figure 9 Power spectrum analysis of the intensity fluctuations of J0137+3309 (3C048 in the 3C Cambridge catalogue) detected by MEXART on 22 April 2009. The 'on-source' spectrum after the subtraction of the system noise: (left) $\log$ - linear scale and (right) $\log -\log$ scale.

is basically the weak $\mathrm{S} / \mathrm{N}$ ratio given by the instrument, this ratio will improve significantly when MEXART is fully operational.

\section{Summary}

The main objective of MEXART is to perform studies of solar-wind disturbances employing the IPS technique. We show the theoretical calculations of the array sensitivity and confusion limit for different configurations. We report the first list of IPS sources detected by the instrument and the spectral analysis of the intensity fluctuations. The observations of IPS using MEXART will allow us a better tracking of solar-wind disturbances, complementing the data provided by the other instruments.

\subsection{Future Work}

The array is under calibration to improve the $\mathrm{S} / \mathrm{N}$ ratio and the directivity of its beams, which will improve the results of the power spectral analysis of the IPS sources. We are tracking these IPS sources to determine the $m$ versus $\epsilon$ curves at $140 \mathrm{MHz}$. We will use these yearly records to infer the $g$ index associated with every radio source. We will produce maps of solar-wind density disturbances. We will apply the single station technique (Manoharan and Ananthakrishnan, 1990) to estimate solar-wind speeds at $140 \mathrm{MHz}$. The fitting model to MEXART observations will allow us to produce solar-wind velocity maps.

Acknowledgements This project was partially supported by CONACyT 48494 and DGA-PA PAPIIT IN105310-3 and IN110309-2 projects. IN110309-2 projects. J.C. Mejia-Ambriz and P. Villanueva-Hernandez thank CONACyT for their Ph.D. grants. We are grateful to Ernesto Andrade, Armando Carrillo, Pablo Sierra, Samuel Vazquez, and Gilberto Casillas for the technical support at MEXART. We are also grateful to P.K. Manoharan of the Ooty Radio Telescope (ORT), Radio Astronomy Centre, TIFR-NCRA, India, and M. Tokumaru of the Solar-Terrestrial Environment Laboratory (STEL/STELab), University of Nagoya, Japan, for providing an updated IPS source list of ORT and STEL observations. We thank the National Centre for Radio Astrophysics (NCRA), Tata Institute of Fundamental Research (TIFR), India, and the Instituto de Geofisica y Astronomia, CITMA, Cuba, for their technical assistance. This research has made use of the NASA/IPAC Extragalactic Database (NED) which is operated by the Jet Propulsion Laboratory, California Institute of Technology, under contract with the National Aeronautics and Space Administration; and the SIMBAD database, operated at CDS, Strasbourg, France. 
Open Access This article is distributed under the terms of the Creative Commons Attribution Noncommercial License which permits any noncommercial use, distribution, and reproduction in any medium, provided the original author(s) and source are credited.

\section{References}

Ananthakrishnan, S., Kaufman, J.: 1980, Microturbulence in solar wind streams microturbulence in solar wind streams. J. Geophys. Res. 85, 6025-6030.

Carrillo, A.: 2007, Construccion y calibracion del radiotelescopio de centelleo interplanetario. Ph.D. thesis, Posgrado en Ciencias de la Tierra, Universidad Nacional Autonoma de Mexico.

Condon, J.: 1974, Confusion and flux-density error distributions. Astrophys. J. 188, 279-286.

Edge, D.O., Shakeshaft, J.R., McAdam, W.B., Baldwin, J.E., Archer, S.: 1959, A survey of radio sources at a frequency of $159 \mathrm{mc} / \mathrm{s}$. Mon. Not. Roy. Astron. Soc. 68, 37-60.

González-Esparza, J.A., Carrillo, A., Andrade, E., Perez-Enriquez, R., Kurtz, S.: 2004, The MEXART interplanetary scintillation array in Mexico. Geofis. Int. 43, 61-73.

González-Esparza, J.A., Andrade, E., Carrillo, A., Jeyakumar, S., Ananthakrishnan, S., Sankarasubramanian, G., Sureshkumar, S., Sierra, P., Vazquez, S., Perez-Enriquez, R., Kurtz, S.: 2005, Mexart measurements of radio sources. In: Solar Wind 11/SOHO 16, Connecting Sun and Heliosphere SP-592, ESA, Noordwijk, $667-669$.

González-Esparza, J.A., Carrillo, A., Andrade, E., Sierra, P., Vazquez, S., Perez-Enriquez, R., Kurtz, S., Blanco-Cano, X.: 2006, Calibration and testing of the MEXART antenna using solar transits. Adv. Space Res. 38, $1824-1827$.

Hewish, A., Bravo, S.: 1986, The sources of large-scale heliospheric disturbances. Solar Phys. 106(1), 185200.

Hewish, A., Scott, P., Wills, D.: 1964, Interplanetary scintillation of small diameter radio sources. Nature 203(495), 1214.

Krauss, J.D.: 1986, Radio Astronomy, 2nd edn., Cygnus-Quasar Books, Powell. ISBN 1-882484-00-2.

Manoharan, P.: 1991, Study of solar wind using single-station interplanetary scintillation. Ph.D. thesis, Bombay University, India.

Manoharan, P.K., Ananthakrishnan, S.: 1990, Determination of solar-wind velocities using single-station measurements of interplanetary scintillation. Mon. Not. Roy. Astron. Soc., 691-695.

Manoharan, P., Ananthakrishnan, S., Dryer, M., Detman, T., Leinbach, H., Kojima, M., Watanabe, T., Kahn, J.: 1995, Solar wind velocity and normalized scintillation index from single-station IPS observations. Solar Phys. 16, 377 - 393.

Oberoi, D.: 2000, Tomography of the solar wind using interplanetary scintillation. Ph.D. thesis, Indian Institute of Science, India.

Pozar, D.M.: 2002, Pcaad 5.0: Personal Computer Aided Antenna Design, Antenna Design Associates, Leverett.

Rao, A.P., Bhandari, S., Ananthakrishnan, S.: 1974, Observations of interplanetary scintillations at $327 \mathrm{MHz}$. Aust. J. Phys. 27(1), $105-120$.

Shankarsubramanian, G., Sureshkumar, S.: 2003, Beam former and data processing system for the Mexican IPS array. Technical Report Mxa/tr/2k3-02, GMRT-TIFR, India. 